\title{
Mycobacterium fallax sp. nov.
}

\author{
VÉRONIQUE LÉVY-FRÉBAULT, ${ }^{1 *}$ ÉLIE RAFIDINARIVO, ${ }^{2}$ JEAN-CLAUDE PROMÉ, ${ }^{2}$ JEANNINE \\ GRANDRY,${ }^{1}$ HENRI BOISVERT,${ }^{1}$ AND HUGO L. DAVID ${ }^{1}$
}

Unité de la Tuberculose et des Mycobactéries, Institut Pasteur, 75724 Paris Cedex $15,{ }^{1}$ and Centre de Biochimie et Génétique Cellulaire du Centre National de la Recherche Scientifique associé à l'Université Paul Sabatier, 31062 Toulouse Cedex, ${ }^{2}$ France

\begin{abstract}
A new species, Mycobacterium fallax, is described. A study of 22 strains showed that they form a homogeneous group with an internal phenotypic similarity value of $94.6 \pm 4.1 \%$. The colony morphology resembled that of Mycobacterium tuberculosis, with cord formation on solid medium. The characteristic features of these strains were that they were nonchromogenic and grew rapidly at $30^{\circ} \mathrm{C}$ but slowly at $37^{\circ} \mathrm{C}$. Most formed a thermolabile catalase and produced nitrate reductase. A lipid analysis showed that tuberculostearic acid was present and that only $\alpha$-mycolic acids were formed. These $\alpha$-mycolic acids were di- and tri-unsaturated acids, a feature that has not been described previously in the mycobacteria. The type strain has been deposited in the Collection Nationale de Cultures de Microorganismes, Paris, France, as strain CIP 8139.
\end{abstract}

The properties of 22 mycobacterial strains that form a homogeneous cluster distinct from the previously known Mycobacterium species are described in this paper. These organisms were characterized for 47 conventional taxonomic characteristics $(10,17)$. Because lipid analysis has been useful in resolving taxonomic problems in the genus Mycobacterium $(1,14)$, the lipid compositions of these strains were also investigated. The strains which we studied formed characteristic mycolic acids, including a new mycolic acid structure.

\section{MATERIALS AND METHODS}

Bacterial strains. A total of 22 strains were studied. Three of these were isolated from sputum, 14 were isolated from water samples from the River Seine between Melun and Conflans-Sainte-Honorine, 2 were isolated from water samples from the River Marne, a tributary of the River Seine, and 1 was isolated from Lake Settons, which is $250 \mathrm{~km}$ from Paris, France. The remaining two strains were kindly sent to us by $\mathbf{M}$. Kubín (Institute of Hygiene and Epidemiology, Prague, Czechoslovakia), who isolated them from soil samples in a region of Czechoslovakia where $\mathrm{Myco}$ bacterium kansasii is common (9). In addition to these 22 isolates, the following strains of species which are on the Approved Lists of Bacterial Names (19) were studied: Mycobacterium chelonei NCTC $946^{\mathrm{T}}$ (type strain); Mycobacterium chitae ATCC $19627^{\mathrm{T}}$; Mycobacterium farcinogenes NCTC $10955^{\mathrm{T}}$; Mycobacterium flavescens ATCC $14474^{\mathrm{T}}$; Mycobacterium fortuitum ATCC $6841^{\mathrm{T}}$; Mycobacterium gastri ATCC $15754^{\mathrm{T}} ;$ Mycobacterium gordonae ATCC $14470^{\mathrm{T}} ; M y-$ cobacterium kansasii ATCC $12478^{\mathrm{T}}$; Mycobacterium marinum ATCC $927^{\mathrm{T}}$; Mycobacterium senegalense NCTC $10956^{\mathrm{T}}$; Mycobacterium smegmatis ATCC $19420^{\mathrm{T}}$; Mycobacterium terrae ATCC $15755^{\mathrm{T}}$; Mycobacterium triviale ATCC $23292^{\mathrm{T}} ;$ Mycobacterium thermoresistibile ATCC $19527^{\mathrm{T}}$; Mycobacterium tu- berculosis ATCC $27294^{\mathrm{T}}$; and Mycobacterium vaccae ATCC $15483^{\mathrm{T}}$. In addition, the frequency distribution of the test results for 608 mycobacterial isolates from human hosts were included for comparative purposes. These isolates included $31 \mathrm{M}$. chelonei strains, $14 \mathrm{M}$. flavescens strains, $63 \mathrm{M}$. fortuitum strains, $43 \mathrm{M}$. gastri strains, $99 M$. gordonae strains, 58 M. kansasii strains, $14 M$. marinum strains, $47 M$. terrae strains, and $239 M$. tuberculosis strains.

Characterization of strains. The niacin test was performed with test strips (Difco Laboratories, Detroit, Mich.). The catalase test was done by the method of Kubica and Pool (11), and the nitrate reductase and arylsulfatase tests were performed as described by Vestal (22). For the Tween hydrolysis test, we used a commercial substrate obtained from Difco. The urease and $\beta$-glucosidase tests were performed by the methods of Meyer and David (13) and David and Jahan (5), respectively. The penicillinase and trehalase tests were performed by following the recommendations of David et al. (6). The iron uptake test and the three tests concerning growth on inositol, mannitol, and sodium citrate were done as described by Silcox et al. (18). The medium for testing growth on fructose was prepared as described by Gordon and Smith (8). The medium for testing the growth on hydroxylamine contained $250 \mu \mathrm{g}$ of hydroxylamine per $\mathrm{ml}$ (21). Growth on the different antibiotics tested was determined by the method of Canetti et al. (4). For the cephalosporinase test, the substrate was prepared by dissolving $5 \mathrm{mg}$ of Nitrocefin in $0.5 \mathrm{ml}$ of dimethyl

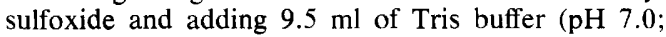
Sigma Chemical Co., St. Louis, Mo.). This solution was distributed in $0.5-\mathrm{ml}$ amounts into screw-capped test tubes ( 16 by $125 \mathrm{~mm}$ ). About $5 \mathrm{mg}$ of cells scraped from the surface of Löwenstein-Jensen slants was suspended in each substrate solution, and the mixtures were incubated at $37^{\circ} \mathrm{C}$ for $2 \mathrm{~h}$. The development of a distinct red color indicated a positive reaction.

Cluster analysis. The data obtained from the study of 47 taxonomic properties were converted to the simple 
binary form (i.e., 1 or 0 ). The degree of internal similarity of the 22 strains of Mycobacterium fallax $\mathrm{sp}$. nov. was determined by using the simple matching coefficient (20). Using the same method, we compared the $M$. fallax type strain with the type strains of 16 previously described mycobacterial species.

Cultivation of strains. Most of the bacteria were grown in Löwenstein-Jensen medium at $37^{\circ} \mathrm{C}$; exceptions were the $M$. fallax strains, which were cultivated at $30^{\circ} \mathrm{C}$. For the isolation of lipid components, bacteria were cultivated on Middlebrook 7 H9 base (Difco) supplemented with $0.5 \%$ (wt/vol) Casitone and $1 \%$ (wt/vol) glucose. Cultures were stirred or shaken during growth and harvested by centrifugation after 8 days at $30^{\circ} \mathrm{C}$ (M. fallax strains) or after 15 days at $37^{\circ} \mathrm{C}$ (M. triviale type strain).

Isolation, purification, and analysis of the lipids. The lipid analysis procedure used for the $M$. fallax strains and the $M$. triviale type strain was that recommended by Asselineau et al. (3). Argentation chromatography was performed by the method of Mangold (12), and visualization of the spots was obtained by charring after spraying with sulfuric acid.

Instrumentation. Gas chromatographic profiles were obtained with a Girdel gas chromatograph by using a $3 \%$ OV1 stationary phase on Chromosorb W (column dimensions, $3 \mathrm{~m}$ by $1 / 8$ in. [3.2 mm]). For pyrolytic gas chromatography, the injector temperature was raised from 220 to $300^{\circ} \mathrm{C}$. Mass spectrometric measurements were done with a Varian model MAT 311A instrument. The electron energy was $70 \mathrm{eV}$, the source temperature was $250^{\circ} \mathrm{C}$, and the direct probe temperature was about $280^{\circ} \mathrm{C}$.

Phage studies. The $M$. fallax strains were examined by the methods of Rado et al. (15), except that the inoculated plates were incubated at $30^{\circ} \mathrm{C}$ for $3 \mathrm{~h}$ before they were spotted with phages. Cultures were examined for phage lysis 1 and 5 days after spotting. The following phage titers were used: DS6A, $4 \times 10^{8}$ plaque-forming units (PFU); BK1, $2 \times 10^{10} \mathrm{PFU}$; BG1, $5 \times 10^{6}$ PFU; D-29, $2 \times 10^{10} \mathrm{PFU}$; and D-33, $3 \times$ $10^{8}$ PFU. The plate medium used was Rva29 (16), which was used without the addition of albumin.

\section{RESULTS AND DISCUSSION}

Cells of the 22 previously undescribed strains studied were acid-fast short rods which formed high-molecular-weight $\left(\mathrm{C}_{70}\right.$ to $\left.\mathrm{C}_{78}\right)$ mycolic acids and were susceptible to mycobacteriophage D29 . These properties were taken as evidence for the inclusion of these strains in the genus $\mathrm{Myco-}$ bacterium.

A phenotypic similarity value of $94.6 \pm 4.1 \%$ among the 22 strains showed their high degree of homogeneity and allowed the differentiation of these strains from other mycobacterial species as a distinct group. This group is formally described below as $M$. fallax sp. nov.

Comparisons of $M$. fallax CIP $8139^{\mathrm{T}}$ with the type strains of 16 mycobacterial species showed degrees of similarity of $72.34 \%$ with $M$. farcinogenes, $71.74 \%$ with $M$. marinum, and $70.21 \%$ with $M$. triviale (Table 1).

The main differences between $M$. fallax and $M$. farcinogenes were the following characteris-
TABLE 1 . Similarity between $\boldsymbol{M}$. fallax strain CIP $8139^{\mathrm{T}}$ and type strains of 16 other mycobacterial species

\begin{tabular}{|c|c|}
\hline Species & $\%$ Similarity to CIP $8139^{\mathrm{T}}$ \\
\hline M. farcinogenes. . & 72.34 \\
\hline M. marinum ${ }^{a} \ldots \ldots \ldots \ldots$ & 71.74 \\
\hline M. triviale ............. & 70.21 \\
\hline M. gastri ............ & 68.08 \\
\hline$M$. gordonae............. & 68.08 \\
\hline M. kansasii. ............ & 68.08 \\
\hline$M$. senegalense $\ldots \ldots \ldots \ldots \ldots$ & 68.08 \\
\hline M. chitae............ & 63.83 \\
\hline M. smegmatis ........... & 63.83 \\
\hline$M$. terrae............... & 63.83 \\
\hline M. tuberculosis . .......... & 63.83 \\
\hline M. vaccae ............ & 63.83 \\
\hline M. fortuitum $\ldots \ldots \ldots \ldots \ldots$ & 57.45 \\
\hline M. flavescens . . . . . . . . & 55.32 \\
\hline M. thermoresistibile ....... & 55.32 \\
\hline M. chelonei ${ }^{a} \ldots \ldots \ldots \ldots \ldots$ & 50.00 \\
\hline
\end{tabular}

${ }^{a}$ Only 46 properties were compared.

tics of $M$. fallax: growth in less than 5 days at $30^{\circ} \mathrm{C}$, positive nitrate reductase test, negative $\beta$ glucosidase and cephalosporinase tests, utilization of fructose as a sole carbon source, and susceptibility to ethambutol and cycloserine. $M$. fallax strains were easily differentiated from $M$. marinum by the absence of photochromogenicity, a positive nitrate reductase test, negative urease and cephalosporinase tests, and resistance to ethionamide and pyrazinamide. $M . f a l$ lax strains differed from $M$. triviale by producing growth in less than 5 days at $30^{\circ} \mathrm{C}$, having a thermolabile catalase, showing negative reactions for arylsulfatase, trehalase, and urease, utilizing fructose as a sole carbon source, and being unable to grow on $5 \% \mathrm{NaCl}$.

The $M$. fallax strains could be confused with $M$. tuberculosis on the basis of colonial morphology. In addition, they were weakly catalase positive, their catalase was thermolabile, and they were nitrate reductase positive, as $M$. $t u$ berculosis is. However, the $M$. fallax strains were niacin and $\beta$-glucosidase negative.

Phage studies. M. fallax strains were lysed by phage D-29 after 1 day of incubation. Concentrated phage D-29 produced either complete lysis or confluent plaques. However, when phage D-29 lysate was diluted 10 times, isolated plaques were not obtained. This could be attributed to the extreme roughness of the $M$. fallax cultures, which caused difficulties in reading. All of the strains except one were resistant to the other phages tested; the exception was 1 of the 22 strains which was susceptible to phage DS6A.

Results after 5 days of incubation generally confirmed the observations described above. Lysis caused by phage D-29 was more apparent, but only two strains showed isolated plaques 
with the 10-fold-diluted lysate. No lysis occurred with the remaining phages, except for two strains which were susceptible to phage DS6A, one strain which was susceptible to phage $\mathrm{BK1}$, and another one strain that was susceptible to phage BG1.

Lipid chromatographic profiles. The lipid fraction obtained after saponification of $M$. fallax whole cells was separated by thin-layer chromatography before and after methyl ester formation. In addition to common fatty acid esters, $\alpha-$ mycolic acid esters were observed. $\beta$ - and $\gamma$ mycolic and dicarboxylic mycolic acid esters were not detected. A gas chromatographic analysis of the total fatty acid methyl esters indicated the presence of myristic acid $\left(\mathrm{C}_{14: 0}\right)$, palmitoleic acid $\left(\mathrm{C}_{16: 1}\right)$, palmitic acid $\left(\mathrm{C}_{16: 0}\right.$, major component $)$, oleic acid $\left(\mathrm{C}_{18: 1}\right.$, major component), stearic acid $\left(\mathrm{C}_{18: 0}\right)$, and tuberculostearic acid $\left(\mathrm{rC}_{19}\right)$. An unidentified peak was detected close to the $\mathrm{C}_{17}$ acid peak. When pyrolysis gas chromatography was used (injector temperature, $300^{\circ} \mathrm{C}$ ), intense peaks corresponding to $\mathrm{C}_{22}$ and $\mathrm{C}_{24}$ fatty acid esters were observed, indicating the presence of $\alpha$-branched, $\beta$-hydroxylated esters of the mycolic acid type containing $n-C_{20}$ and $n-C_{22}$ aliphatic $\alpha$-branching.

Mass spectrometric analysis of the $\alpha$-mycolic methyl esters. After purification, the methyl esters of the $\alpha$-mycolic acids were analyzed by mass spectrometry under electron impact. The total numbers of carbon atoms and the numbers of unsaturations or cycles were deduced from the mass/charge values of the (M-50) ${ }^{+}$ions, which were formed by successive losses of water and methanol from the molecular ion $\mathrm{M}^{+}$. The carbon content ranged between 70 and 78

TABLE 2. Numbers of carbon atoms and unsaturations or cycles of the $M$. fallax $\alpha$-mycolic acids deduced from the $(\mathrm{M}-50)^{+\cdot}$ ions

\begin{tabular}{cc}
\hline $\begin{array}{c}\text { Mass/charge value of } \\
(\mathrm{M}-50)^{+*} \text { ion }^{a}\end{array}$ & $\begin{array}{c}\text { No. of carbon atoms } \\
\text { and unsaturations }\end{array}$ \\
\hline 1,100 & $78: 2^{b}$ \\
1,098 & $78: 3$ \\
1,086 & $77: 2$ \\
1,072 & $76: 2$ \\
1,070 & $76: 3$ \\
1,058 & $75: 2$ \\
1,044 & $74: 2$ \\
1,042 & $74: 3$ \\
1,030 & $73: 2$ \\
1,016 & $72: 2$ \\
1,002 & $71: 2$ \\
998 & $70: 2$ \\
\hline
\end{tabular}

${ }^{a}$ The $(\mathrm{M}-50)^{+\cdot}$ ions correspond to the $\mathrm{M}^{+\cdot}$ ions after successive losses of one methanol molecule and one water molecule.

$b$ The number to the left of the colon indicates the number of carbon atoms, and the number to the right of the colon indicates the number of double bonds or cyclopropanes.
TABLE 3. Major ions corresponding to the aldehydes obtained after pyrolysis of the $M$. fallax $\alpha$ mycolic acids

\begin{tabular}{cc}
\hline $\begin{array}{c}\text { Mass/charge value of } \\
(\mathbf{M}-50)^{+*} \text { ion }\end{array}$ & $\begin{array}{c}\text { No. of carbon atoms } \\
\text { and unsaturations }\end{array}$ \\
\hline 782 & $55: 2^{a}$ \\
768 & $54: 2$ \\
766 & $54: 3$ \\
754 & $53: 2$ \\
740 & $52: 2$ \\
738 & $52: 3$ \\
726 & $51: 2$ \\
712 & $50: 2$ \\
\hline
\end{tabular}

${ }^{a}$ The number to the left of the colon indicates the number of carbon atoms, and the number to the right of the colon indicates the number of double bonds or cycles.

atoms (Table 2). The splitting of the mycolates into aldehydes and esters produced the ions shown in Table 3. Peaks occurring at mass/ charge values of 354 and 382 were due to the $\mathrm{C}_{22: 0}$ and $\mathrm{C}_{24: 0}$ acid methyl esters ions; this confirmed the gas chromatographic observations. The peak values corresponding to the meroaldehyde ions indicated that all of the unsaturations or cycles were located in the meromycolic fragment (Table 3).

Mycolic acid analysis according to the degree of unsaturation. To determine the nature of the meromycolic chain functions (i.e., unsaturations or cycles), the $\alpha$-mycolate fraction was separated on Silica Gel G impregnated with $5 \%(\mathrm{wt} / \mathrm{wt})$ silver nitrate (12). Three major spots were observed and compared with authentic samples. A mass spectrometric analysis of each component allowed us to determine hydrogenation content. The following components were observed (with decreasing $R_{f}$ values): di-unsaturated $\alpha$-mycolate I, di-unsaturated $\alpha$-mycolate II, and triunsaturated $\alpha$-mycolate.

The di-unsaturated $\alpha$-mycolate II possessed the same $R_{f}$ value on silver nitrate plates as an $M$. smegmatis $\alpha$-mycolate, in which the two cis unsaturations of the meromycolate chain were surrounded only by unbranched polymethylenic chains (7). The behavior of $\alpha$-mycolate I resembled that of the other $\alpha$-mycolate acids from $M$. smegmatis, in which one carbon-carbon double bond having a trans configuration possessed a methyl branching on an aldehyde position (7). The tri-unsaturated mycolic acid of $\boldsymbol{M}$. fallax represents a new type of mycolic acid. This characteristic proved to be decisive for differentiation of $M$. fallax and $M$. triviale strains. Mycolic acids containing more than two double bonds or two cyclopropanes have not been described previously $(2,14)$. Tri-unsaturation in hydrocarbons having different chain lengths has been reported in Nocardia and Rhodococcus species (14).

Since the $M$. fallax strains and $M$. triviale ATCC $23292^{\mathrm{T}}$ produced similar lipid profiles 


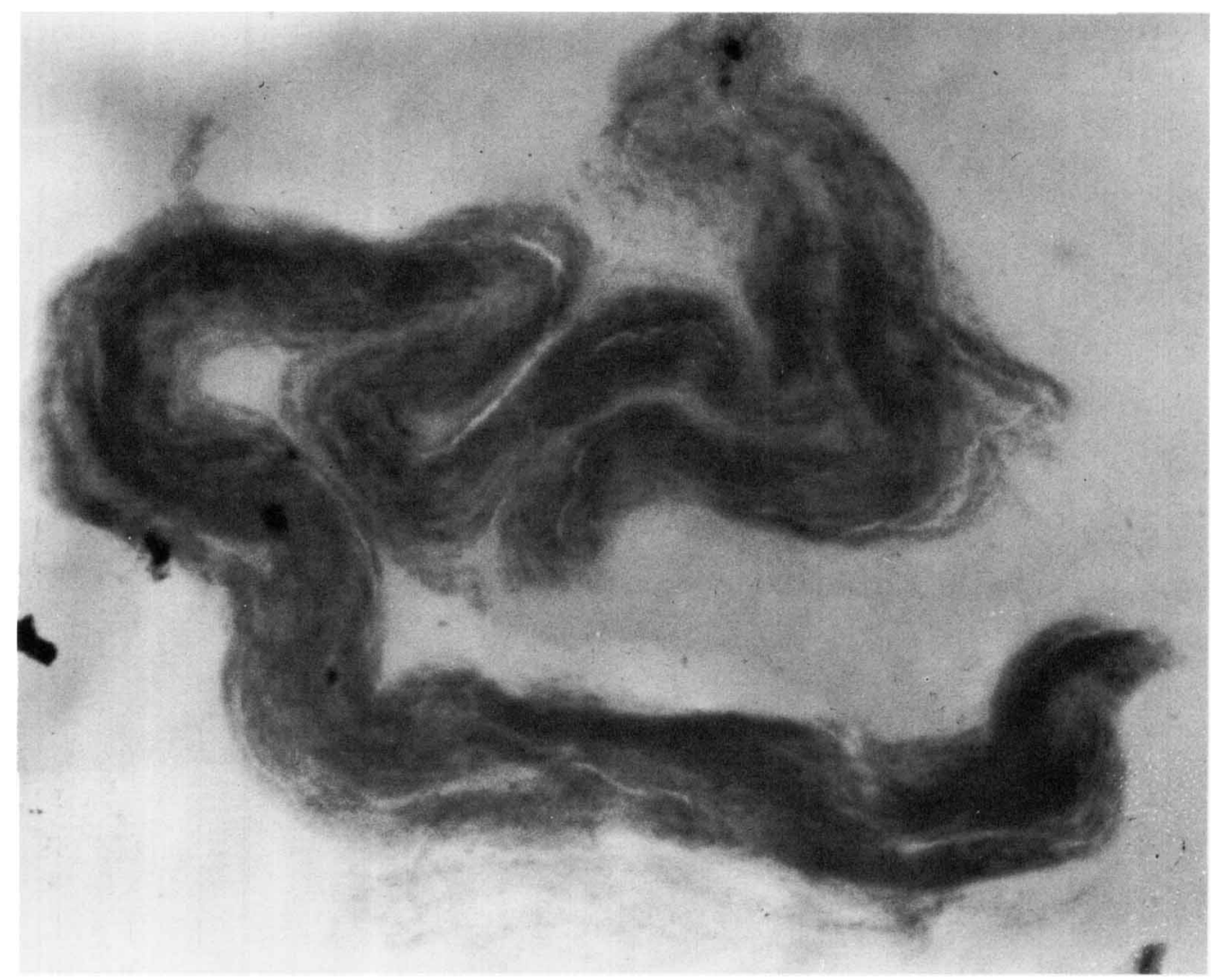

FIG. 1. Smears prepared from $M$. fallax grown on Youmans medium, showing cord formation. $\times 40$. Youmans medium was prepared with no Tween 80.
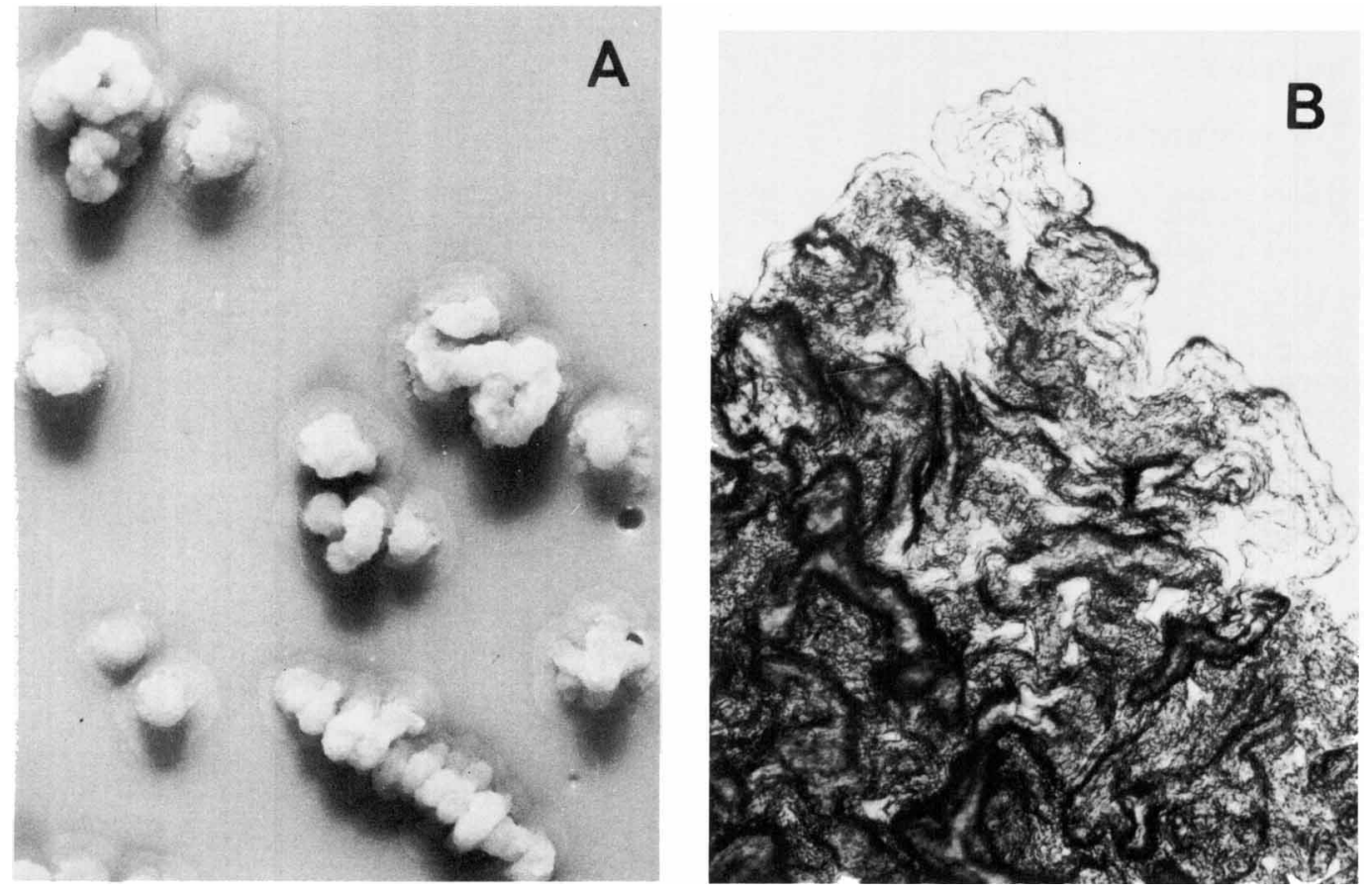

FIG. 2. M. fallax CIP $8139^{\mathrm{T}}$ colonies on solid medium. (A) Löwenstein-Jensen medium. No magnification. (B) Middlebrook $7 \mathrm{H} 10$ medium. $\times 6$. 
TABLE 4. Properties of $M$. fallax and other mycobacteria

\begin{tabular}{|c|c|c|c|c|c|c|c|c|c|c|c|c|}
\hline \multirow[b]{2}{*}{ Characteristic } & \multicolumn{2}{|c|}{ M. fallax } & \multicolumn{2}{|c|}{ M. chelonei } & \multirow{2}{*}{$\begin{array}{c}M . \\
\text { chitae } \\
\text { type } \\
\text { strain }\end{array}$} & \multirow{2}{*}{$\begin{array}{c}\text { M. farci- } \\
\text { nogenes } \\
\text { type strain }\end{array}$} & \multicolumn{2}{|c|}{ M. flavescens } & \multicolumn{2}{|c|}{ M. fortuitum } & \multicolumn{2}{|c|}{ M. gastri } \\
\hline & $\begin{array}{c}\text { Type } \\
\text { strain }\end{array}$ & $\begin{array}{c}22 \\
\text { Other } \\
\text { strains }\end{array}$ & $\begin{array}{l}\text { Type } \\
\text { strain }\end{array}$ & $\begin{array}{c}31 \\
\text { Other } \\
\text { strains }\end{array}$ & & & $\begin{array}{l}\text { Type } \\
\text { strain }\end{array}$ & $\begin{array}{c}14 \\
\text { Other } \\
\text { strains }\end{array}$ & $\begin{array}{l}\text { Type } \\
\text { strain }\end{array}$ & $\begin{array}{c}63 \\
\text { Other } \\
\text { strains }\end{array}$ & $\begin{array}{l}\text { Type } \\
\text { strain }\end{array}$ & $\begin{array}{c}43 \\
\text { Other } \\
\text { strains }\end{array}$ \\
\hline Rods $(>1 \mu \mathrm{m})$ & $-{ }^{a}$ & 0 & + & ND & - & + & + & 100 & + & 100 & + & 100 \\
\hline $\begin{array}{l}\text { Coccobacillary } \\
(<1 \mu \mathrm{m})\end{array}$ & + & 100 & - & ND & + & - & - & 0 & - & 0 & - & 0 \\
\hline Cord formation & + & 100 & + & ND & - & - & - & ND & - & ND & - & ND \\
\hline Colonies smooth & - & 0 & + & ND & + & - & - & ND & + & $\mathrm{ND}$ & + & ND \\
\hline Colonies rough & + & 100 & - & ND & - & + & + & ND & - & ND & - & ND \\
\hline \multicolumn{13}{|l|}{ Growth at: } \\
\hline $30^{\circ} \mathrm{C}$ & + & 100 & + & 100 & + & + & + & 100 & + & 100 & + & 100 \\
\hline $30^{\circ} \mathrm{C},<5$ days & + & 100 & + & 100 & + & - & - & ND & + & 100 & - & 0 \\
\hline $37^{\circ} \mathrm{C}$ & + & 77 & + & 100 & + & + & + & 100 & + & 100 & + & 100 \\
\hline $37^{\circ} \mathrm{C},<5$ days & - & 18 & ND & ND & + & - & - & 0 & + & 100 & - & 0 \\
\hline $45^{\circ} \mathrm{C}$ & - & 0 & - & 0 & - & - & - & 0 & - & 0 & - & 0 \\
\hline $52^{\circ} \mathrm{C}$ & - & 0 & - & 0 & - & - & - & 0 & - & 0 & - & 0 \\
\hline \multicolumn{13}{|l|}{$\begin{array}{l}\text { Growth on nutrient } \\
\text { agar at: }\end{array}$} \\
\hline $30^{\circ} \mathrm{C}$ & + & 100 & + & 100 & + & - & + & ND & + & ND & - & ND \\
\hline $37^{\circ} \mathrm{C}$ & - & 18 & + & ND & + & - & + & 100 & + & 100 & - & 0 \\
\hline $\begin{array}{l}\text { Pigmentation in the } \\
\text { dark }\end{array}$ & - & 0 & - & 0 & - & - & + & 100 & - & 0 & - & 0 \\
\hline $\begin{array}{l}\text { Pigmentation in the } \\
\text { light }\end{array}$ & - & 0 & - & 0 & - & - & + & 100 & - & 0 & - & 0 \\
\hline Niacin & - & 0 & - & 0 & - & - & - & 0 & - & 0 & - & 0 \\
\hline \multicolumn{13}{|l|}{ Enzymatic activity: } \\
\hline $\begin{array}{l}\text { Arylsulfatase } \\
\text { (3 days) }\end{array}$ & - & 0 & + & 100 & - & - & + & 86 & + & 100 & - & ND \\
\hline $\begin{array}{l}\text { Catalase } \\
\left(22^{\circ} \mathrm{C}\right)\end{array}$ & + & 100 & + & 100 & + & + & + & 100 & + & 100 & + & 100 \\
\hline $\begin{array}{r}\text { Catalase } \\
\left(68^{\circ} \mathrm{C}\right)\end{array}$ & - & 27 & + & 84 & + & + & + & 100 & + & 98 & - & 0 \\
\hline $\begin{array}{l}\text { Cephalosporinase } \\
(2 \mathrm{~h})\end{array}$ & - & $11^{b}$ & + & 100 & + & + & + & 33 & + & 100 & + & 100 \\
\hline $\begin{array}{l}\beta \text {-Glucosidase } \\
(3 \mathrm{~h})\end{array}$ & - & 0 & - & 0 & - & - & - & 7 & + & 100 & - & 82 \\
\hline $\begin{array}{l}\text { Nitrate reductase } \\
(2 \mathrm{~h})\end{array}$ & + & 100 & - & 0 & + & - & + & 100 & + & 100 & - & 0 \\
\hline Penicillinase $(5 \mathrm{~h})$ & - & 0 & + & 100 & - & - & - & 14 & - & 0 & - & 3 \\
\hline Trehalase $(5 \mathrm{~h})$ & - & 9 & + & 100 & - & - & - & 7 & - & 0 & - & 16 \\
\hline $\begin{array}{l}\text { Tween hydrolysis } \\
\text { (10 days) }\end{array}$ & + & 100 & + & 90 & - & + & + & 100 & + & 83 & + & 100 \\
\hline Urease $(18 \mathrm{~h})$ & - & 4 & + & 26 & + & - & + & 14 & + & 24 & + & 5 \\
\hline Iron uptake & - & 0 & + & ND & + & - & - & ND & + & ND & - & ND \\
\hline $\begin{array}{l}\text { Growth on MacConkey } \\
\text { agar }\end{array}$ & - & 0 & + & ND & - & - & - & ND & + & ND & - & ND \\
\hline \multicolumn{13}{|l|}{$\begin{array}{l}\text { Use as sole carbon } \\
\text { source: }\end{array}$} \\
\hline Fructose & + & 100 & - & 0 & + & - & - & ND & + & 100 & - & 0 \\
\hline Inositol & - & 0 & - & ND & + & - & - & ND & - & ND & - & ND \\
\hline Mannitol & - & 0 & - & ND & + & - & + & ND & - & ND & - & ND \\
\hline Sodium citrate & - & 0 & + & ND & - & - & - & ND & - & ND & - & ND \\
\hline \multicolumn{13}{|l|}{$\begin{array}{l}\text { Growth in the presence } \\
\text { of: }\end{array}$} \\
\hline $5 \% \mathrm{NaCl}$ & - & 0 & - & ND & + & - & + & ND & + & ND & - & ND \\
\hline $\begin{array}{l}\mathrm{NH}_{2} \mathrm{OH} \\
\quad(250 \mu \mathrm{g} / \mathrm{ml})\end{array}$ & + & 100 & + & 100 & + & - & - & 21 & + & 100 & + & 100 \\
\hline $\begin{array}{l}\text { Amithiazone } \\
\qquad(2 \mu \mathrm{g} / \mathrm{ml})\end{array}$ & + & 100 & + & 100 & + & + & + & 100 & + & 100 & - & 0 \\
\hline $\begin{array}{l}\text { Capreomycin } \\
\qquad(40 \mu \mathrm{g} / \mathrm{ml})\end{array}$ & - & 0 & + & ND & - & - & - & ND & - & ND & - & ND \\
\hline $\begin{array}{l}\text { D-Cycloserine } \\
(30 \mu \mathrm{g} / \mathrm{ml})\end{array}$ & - & 0 & + & ND & + & + & + & ND & + & ND & - & ND \\
\hline
\end{tabular}


TABLE 4-Continued

\begin{tabular}{|c|c|c|c|c|c|c|c|c|c|c|c|c|c|c|}
\hline \multicolumn{2}{|c|}{ M. gordonae } & \multicolumn{2}{|c|}{ M. kansasii } & \multicolumn{2}{|c|}{ M. marinum } & \multirow{2}{*}{$\begin{array}{l}\text { M. senegal- } \\
\text { ense type } \\
\text { strain }\end{array}$} & \multirow{2}{*}{$\begin{array}{c}M . \text { smeg- } \\
\text { matis } \\
\text { type } \\
\text { strain }\end{array}$} & \multicolumn{2}{|c|}{ M. terrae } & \multirow{2}{*}{$\begin{array}{c}M . \\
\text { thermo- } \\
\text { resistibile } \\
\text { type } \\
\text { strain }\end{array}$} & \multirow{2}{*}{$\begin{array}{l}\text { M. trivi- } \\
\text { ale type } \\
\text { strain }\end{array}$} & \multicolumn{2}{|c|}{$\begin{array}{c}M \\
\text { tuberculosis }\end{array}$} & \multirow{2}{*}{$\begin{array}{c}\text { M. vac- } \\
\text { cae type } \\
\text { strain }\end{array}$} \\
\hline $\begin{array}{l}\text { Type } \\
\text { strain }\end{array}$ & $\begin{array}{c}99 \\
\text { Other } \\
\text { strains }\end{array}$ & $\begin{array}{c}\text { Type } \\
\text { strain }\end{array}$ & \begin{tabular}{|c|}
58 \\
Other \\
strains
\end{tabular} & $\begin{array}{l}\text { Type } \\
\text { strain }\end{array}$ & \begin{tabular}{|c|}
14 \\
Other \\
strains
\end{tabular} & & & \begin{tabular}{|l|} 
Type \\
strain
\end{tabular} & $\begin{array}{c}47 \\
\text { Other } \\
\text { strains }\end{array}$ & & & $\begin{array}{l}\text { Type } \\
\text { strain }\end{array}$ & $\begin{array}{c}239 \\
\text { Other } \\
\text { strains }\end{array}$ & \\
\hline+ & 100 & + & 100 & + & 100 & + & + & + & 100 & + & + & + & 100 & - \\
\hline- & 0 & - & 0 & - & 0 & - & - & - & 0 & - & - & - & 0 & + \\
\hline - & ND & + & ND & + & ND & - & - & - & ND & - & + & + & 100 & - \\
\hline+ & 100 & - & ND & + & ND & - & - & + & ND & + & - & - & 0 & + \\
\hline- & 0 & + & ND & - & ND & + & + & - & ND & - & + & + & 100 & - \\
\hline+ & 100 & + & 100 & + & 100 & + & + & + & 100 & + & + & + & 100 & + \\
\hline- & 0 & - & 0 & + & 100 & + & + & - & 0 & + & - & - & 0 & + \\
\hline+ & 100 & + & 100 & ND & ND & + & + & + & 100 & + & + & + & 100 & + \\
\hline- & 0 & - & 0 & - & ND & + & + & - & 0 & + & - & - & 0 & + \\
\hline- & 0 & - & 0 & - & 0 & - & + & - & 0 & + & - & - & 0 & - \\
\hline- & 0 & - & 0 & - & 0 & - & - & - & 0 & + & - & - & 0 & - \\
\hline- & 0 & - & 0 & + & ND & + & + & - & 0 & + & - & - & 0 & + \\
\hline- & 0 & - & 0 & - & ND & + & + & - & 0 & + & - & - & 0 & + \\
\hline+ & 100 & - & 0 & - & 0 & - & - & - & 0 & + & - & - & 0 & + \\
\hline+ & 100 & + & 100 & + & 100 & - & - & - & 0 & + & - & - & 0 & + \\
\hline- & 0 & - & 0 & - & 0 & - & - & - & 0 & - & - & + & 100 & - \\
\hline- & ND & - & 5 & - & ND & + & - & - & ND & - & + & - & ND & - \\
\hline+ & 100 & + & 100 & + & 100 & + & + & + & 100 & + & + & + & 100 & + \\
\hline+ & 100 & + & 100 & + & 100 & + & + & + & 100 & + & + & - & 0 & + \\
\hline+ & 70 & + & 71 & + & 100 & + & + & + & 15 & + & - & + & 96 & + \\
\hline- & 7 & + & 57 & - & 7 & + & - & - & 40 & - & - & + & 90 & + \\
\hline- & 0 & + & 100 & - & 0 & + & + & + & 100 & + & + & + & 100 & + \\
\hline- & 0 & - & 0 & - & 0 & - & + & - & 2 & - & - & - & 5 & - \\
\hline- & 71 & + & 82 & + & 50 & - & - & + & 27 & - & + & + & 61 & - \\
\hline+ & 100 & + & 100 & + & 100 & - & + & + & 100 & + & + & + & ND & + \\
\hline- & 0 & + & 12 & + & 0 & + & + & - & 0 & + & + & + & 72 & - \\
\hline- & ND & - & ND & - & ND & - & + & - & ND & - & - & - & ND & + \\
\hline- & ND & - & ND & - & ND & - & - & - & ND & - & - & - & ND & - \\
\hline- & ND & - & ND & ND & ND & + & + & - & 0 & - & - & - & ND & + \\
\hline- & ND & - & ND & - & ND & - & + & - & ND & - & - & - & ND & - \\
\hline- & ND & - & ND & - & ND & + & - & - & ND & - & - & - & ND & + \\
\hline- & ND & - & ND & - & ND & - & - & - & ND & - & - & - & ND & - \\
\hline- & ND & - & ND & - & ND & - & + & - & ND & + & + & - & ND & - \\
\hline+ & 100 & + & 92 & + & 80 & + & + & + & 92 & - & + & - & 0 & + \\
\hline+ & 98 & - & 0 & + & 100 & + & + & + & 100 & + & + & - & 0 & + \\
\hline- & ND & - & ND & - & ND & - & - & + & ND & - & + & - & ND & - \\
\hline- & ND & - & ND & - & ND & + & + & + & ND & - & - & - & ND & + \\
\hline
\end{tabular}


TABLE 4-Continued

\begin{tabular}{|c|c|c|c|c|c|c|c|c|c|c|c|c|}
\hline \multirow[b]{2}{*}{ Characteristic } & \multicolumn{2}{|c|}{ M. fallax } & \multicolumn{2}{|c|}{ M. chelonei } & \multirow{2}{*}{$\begin{array}{c}M . \\
\text { chitae } \\
\text { type } \\
\text { strain }\end{array}$} & \multirow{2}{*}{$\begin{array}{c}\text { M. farci- } \\
\text { nogenes } \\
\text { type strain }\end{array}$} & \multicolumn{2}{|c|}{ M. flavescens } & \multicolumn{2}{|c|}{ M. fortuitum } & \multicolumn{2}{|c|}{ M. gastri } \\
\hline & $\begin{array}{l}\text { Type } \\
\text { strain }\end{array}$ & $\begin{array}{c}22 \\
\text { Other } \\
\text { strains }\end{array}$ & $\begin{array}{l}\text { Type } \\
\text { strain }\end{array}$ & $\begin{array}{c}31 \\
\text { Other } \\
\text { strains }\end{array}$ & & & $\begin{array}{l}\text { Type } \\
\text { strain }\end{array}$ & \begin{tabular}{|c}
14 \\
Other \\
strains
\end{tabular} & $\begin{array}{l}\text { Type } \\
\text { strain }\end{array}$ & \begin{tabular}{|c}
63 \\
Other \\
strains
\end{tabular} & $\begin{array}{l}\text { Type } \\
\text { strain }\end{array}$ & $\begin{array}{c}43 \\
\text { Other } \\
\text { strains }\end{array}$ \\
\hline Growth in the pres & & & & & & & & & & & & \\
\hline $\begin{array}{c}\text { Ethambutol } \\
(2 \mu \mathrm{g} / \mathrm{ml})\end{array}$ & - & 0 & + & 100 & - & + & - & 0 & + & 97 & - & 0 \\
\hline $\begin{array}{r}\text { Ethionamide } \\
(20 \mu \mathrm{g} / \mathrm{ml})\end{array}$ & + & $94^{b}$ & ND & ND & + & + & - & ND & - & ND & - & ND \\
\hline $\begin{array}{l}\text { Isoniazid } \\
(0.1 \mu \mathrm{g} / \mathrm{ml})\end{array}$ & + & 100 & + & ND & + & + & - & ND & + & ND & + & ND \\
\hline $\begin{array}{l}\text { Kanamycin } \\
(20 \mu \mathrm{g} / \mathrm{ml})\end{array}$ & - & 0 & + & ND & - & - & - & ND & - & ND & + & ND \\
\hline $\begin{array}{l}\text { PAS } \\
\qquad(0.5 \mu \mathrm{g} / \mathrm{ml})^{c}\end{array}$ & + & 100 & + & ND & + & + & + & ND & + & ND & + & ND \\
\hline $\begin{array}{r}\text { Pyrazinamide } \\
(100 \mu \mathrm{g} / \mathrm{ml})\end{array}$ & + & 100 & + & ND & + & + & + & ND & + & ND & + & ND \\
\hline $\begin{array}{l}\text { Rifampin } \\
\quad(40 \mu \mathrm{g} / \mathrm{ml})\end{array}$ & - & $6^{b}$ & + & ND & + & - & + & ND & + & ND & - & ND \\
\hline $\begin{array}{l}\text { Rifamycin } \\
\quad(40 \mu \mathrm{g} / \mathrm{ml})\end{array}$ & - & $6^{b}$ & + & ND & + & + & + & ND & + & ND & - & ND \\
\hline $\begin{array}{l}\text { Streptomycin } \\
(4 \mu \mathrm{g} / \mathrm{ml})\end{array}$ & + & $88^{b}$ & + & ND & - & + & - & ND & + & ND & - & ND \\
\hline $\mathrm{TCH}(2 \mu \mathrm{g} / \mathrm{ml})^{d}$ & + & 100 & + & 100 & + & + & + & 100 & + & 100 & + & 100 \\
\hline
\end{tabular}

${ }^{a}-$, Negative reaction; + , positive reaction; ND, not done. The numbers indicate percentages of strains positive.

${ }^{b}$ Only 17 strains were studied.

c PAS, $p$-Aminosalicylic acid.

${ }^{d} \mathrm{TCH}$, Thiophencarboxylic acid hydrazide.

after gas chromatography and thin-layer chromatography, we made a more precise comparison by using the structural features of the $\alpha$ mycolates. An attempt to isolate $\alpha$-mycolate from $M$. triviale ATCC $23292^{\mathrm{T}}$ by argentation chromatography indicated that no unsaturated material was present. The mass spectrometry analysis indicated the presence of two and three unsaturations or cycles. Therefore, it is very likely that in $M$. triviale the cyclopropanes are included in the merochain, as in the mycolic acids of many species. Thus, although strains of $M$. fallax and $M$. triviale ATCC $23292^{\mathrm{T}}$ had similar lipid compositions and contained only $\alpha$ type mycolic acids, the $M$. triviale strain had only cyclic mycolic acids containing di- and tricycles (probably cis-cyclopropanes), whereas the $M$. fallax strains contained di- and tri-unsaturated $\alpha$-mycolic acids.

For this distinct group of strains we propose the name Mycobacterium fallax sp. nov. (fal'lax. L. adj. fallax deceptive; in the sense that the colonies resemble colonies of $M$. tuberculosis).

Morphological, growth, and biochemical characteristics of $\boldsymbol{M}$. fallax sp. nov. The cells of $M$. fallax are short rods 0.5 to $1 \mu \mathrm{m}$ long; they are acid fast, except for a small number (less than $20 \%$ ) of cyanophil forms. Smears prepared from pellicles in Youmans medium (with no Tween 80) show clumps of rods or cords (Fig. 1).

At $30^{\circ} \mathrm{C}$, growth occurs within 5 days on Löwenstein-Jensen and Middlebrook 7H10 media and on nutrient agar. At $37^{\circ} \mathrm{C}$, however, most strains grow poorly and slowly (12 to 21 days) on the former two media. Some strains (23\%) do not grow even after 28 days. Only $18 \%$ of the strains grow on nutrient agar at $37^{\circ} \mathrm{C}$.

On Löwenstein-Jensen medium at $30^{\circ} \mathrm{C}$, colonies are large, eugonic, buff colored, and rough, with a cauliflower-like morphology resembling that of $M$. tuberculosis colonies (Fig. 2A). On Middlebrook $7 \mathrm{H} 10$ medium at $30^{\circ} \mathrm{C}$, cord formation is evident at the edges of colonies (Fig. 2B). Colonies on both of these media are the same at 37 as at $30^{\circ} \mathrm{C}$, except that they are somewhat smaller.

The phenotypic properties of $22 \mathrm{M}$. fallax strains are shown in Table 4 . The type strain has been deposited in the Collection Nationale de Cultures de Microorganismes, Paris, France, as strain CIP 8139.

\section{ACKNOWLEDGMENTS}

The financial support of the Ministère de l'Environnement et du Cadre de Vie is gratefully acknowledged.

We thank P. W. Muggleton, Glaxo Group Research Ltd., Greenford, United Kingdom, for supplying the Nitrocefin used in the cephalosporinase test. 
TABLE 4-Continued

\begin{tabular}{|c|c|c|c|c|c|c|c|c|c|c|c|c|c|c|}
\hline \multicolumn{2}{|c|}{ M. gordonae } & \multicolumn{2}{|c|}{ M. kansasii } & \multicolumn{2}{|c|}{ M. marinum } & \multirow{2}{*}{$\begin{array}{c}\text { M. senegal- } \\
\text { ense type } \\
\text { strain }\end{array}$} & \multirow{2}{*}{$\begin{array}{c}\text { M. smeg- } \\
\text { matis } \\
\text { type } \\
\text { strain }\end{array}$} & \multicolumn{2}{|c|}{$M$. terrae } & \multirow{2}{*}{$\begin{array}{c}M . \\
\text { thermo- } \\
\text { resistibile } \\
\text { type } \\
\text { strain }\end{array}$} & \multirow{2}{*}{$\begin{array}{l}\text { M. trivi } \\
\text { ale type } \\
\text { strain }\end{array}$} & \multicolumn{2}{|c|}{$\begin{array}{c}M . \\
\text { tuberculosis }\end{array}$} & \multirow{2}{*}{$\begin{array}{l}\text { M. vac- } \\
\text { cae type } \\
\text { strain }\end{array}$} \\
\hline $\begin{array}{c}\text { Type } \\
\text { strain }\end{array}$ & $\begin{array}{c}99 \\
\text { Other } \\
\text { strains }\end{array}$ & $\begin{array}{l}\text { Type } \\
\text { strain }\end{array}$ & $\begin{array}{c}58 \\
\text { Other } \\
\text { strains }\end{array}$ & $\begin{array}{l}\text { Type } \\
\text { strain }\end{array}$ & $\begin{array}{c}14 \\
\text { Other } \\
\text { strains }\end{array}$ & & & $\begin{array}{l}\text { Type } \\
\text { strain }\end{array}$ & $\begin{array}{c}47 \\
\text { Other } \\
\text { strains }\end{array}$ & & & $\begin{array}{l}\text { Type } \\
\text { strain }\end{array}$ & $\begin{array}{c}239 \\
\text { Other } \\
\text { strains }\end{array}$ & \\
\hline- & 2 & - & 0 & - & 0 & + & - & - & 5 & - & + & - & 0 & - \\
\hline+ & ND & - & ND & - & ND & + & + & - & ND & + & - & - & ND & + \\
\hline+ & ND & + & ND & + & ND & + & + & + & ND & + & + & - & ND & - \\
\hline- & ND & + & ND & - & ND & - & - & + & ND & - & - & - & ND & - \\
\hline+ & ND & + & ND & + & ND & + & + & + & ND & + & + & - & ND & + \\
\hline+ & ND & + & ND & + & ND & + & + & + & ND & - & + & - & ND & + \\
\hline+ & ND & - & ND & + & ND & - & + & + & ND & + & - & - & ND & + \\
\hline+ & ND & + & ND & + & ND & + & + & t & ND & + & - & - & ND & + \\
\hline+ & ND & + & ND & + & ND & + & - & + & ND & - & - & - & ND & - \\
\hline+ & 100 & + & 100 & + & 100 & + & + & + & 100 & + & + & + & 100 & + \\
\hline
\end{tabular}

\section{LITERATURE CITED}

1. Asselineau, C., and J. Asselineau. 1978. Lipides spécifiques des mycobactéries. Ann. Microbiol. (Paris) 129A:49-69.

2. Asselineau, C., and J. Asselineau. 1978. Trehalose-containing glycolipids. Prog. Chem. Fats Other Lipids 16:5699.

3. Asselineau, C., S. Clavel, F. Clément, M. Dafié, H. L. David, M. A. Lanéelle, and J. C. Promé. 1981. Constituants lipidiques de Mycobacterium leprae isolé de tatou infecté expérimentalement. Ann. Microbiol. (Paris) 132A:19-30.

4. Canetti, G., N. Rist, and J. Grosset. 1963. Mesure de la sensibilité du bacille tuberculeux aux drogues antibacillaires par la méthode des proportions. Rev. Tuberc. Pneumol. 27:217-272.

5. David, H. L., and M. T. Jahan. 1977. $\beta$-Glucosidase activity in mycobacteria. J. Clin. Microbiol. 13:6-9.

6. David, H. L., I. Traore, and A. Feuillet. 1981. Differential identification of Mycobacterium fortuitum and Mycobacterium chelonei. J. Clin. Microbiol. 5:383-384.

7. Etémadi, A. H., F. Pinte, and J. Markovits. 1967. Nouvelle analyse des acides mycoliques de Mycobacterium smegmatis. Bull. Soc. Chim. Fr, p. 195-199.

8. Gordon, R. E., and M. M. Smith. 1955. Rapidly growing acid-fast bacteria. II. Species description of Mycobacterium fortuitum Cruz. J. Bacteriol. 69:502-507.

9. Kaustová, J., Ž . Olšovský, M. Kubín, O. Zatloukal, M. Pelikán, and V. Hradil. 1981. Endemic occurrence of Mycobacterium kansasii in water supply systems. J. Hyg. Epidemiol. Microbiol. Immunol. 25:24-30.

10. Kubica, G. P., I. Baess, R. E. Gordon, P. A. Jenkins, J. B. G. Kwapinski, C. McDurmont, S. R. Pattyn, H. Saito, V. Silcox, J. L. Stanford, K. Takeya, and M. Tsukamura. 1972. A co-operative numerical analysis of rapidly growing mycobacteria. J. Gen. Microbiol. 73:5570 .

11. Kubica, G. P., and G. L. Pool. 1960. Studies on the catalase activity of acid fast bacilli. I. An attempt to subgroup these organisms on the basis of their catalase activities at different temperatures and pH. Am. Rev. Respir. Dis. 81:387-391.
11a.Lévy-Frébault, V., J. Grandry, and H. L. David. 1982. Evaluation of rapid tests for the identification of mycobacteria. J. Med. Microbiol. 15:575-577.

12. Mangold, H. K. 1969. Aliphatic lipids: argentation chromatography, p. 396-402. In E. Stahl (ed.), Thin-layer chromatography, 2nd ed. Springer-Verlag, Berlin.

13. Meyer, L., and H. L. David. 1979. Evaluation de l'activité uréase et de l'activité $\beta$-glucosidase pour l'identification pratique des mycobactéries. Ann. Microbiol. (Paris) 130B:323-332.

14. Minnikin, D. E., and M. Goodfellow. 1980. Lipid composition in the classification and identification of acid-fast bacteria, p. 189-256. In M. Goodfellow and R. G. Board (ed.), Microbiological classification and identification. Academic Press, Inc., London.

15. Rado, T. A., J. H. Bates, H. W. B. Engel, E. Mankiewicz, T. Murohashi, Y. Mizuguchi, and L. Šula. 1975. World Health Organization studies on bacteriophage typing of mycobacteria. Subdivision of the species Mycobacterium tuberculosis. Am. Rev. Respir. Dis. 111:459-468.

16. Redmond, W. B., and D. M. Ward. 1966. Media and methods for phage typing mycobacteria. Bull. W.H.O. 35:563-568.

17. Saito, H., R. E. Gordon, I. Juhlin, W. Kappler, J. B. G. Kwapinski, C. McDurmont, S. R. Pattyn, E. H. Runyon, J. L. Stanford, I. Tarnok, H. Tasaka, M. Tsukamura, and J. Weiszfeiler. 1977. Cooperative numerical analysis of rapidly growing mycobacteria. Int. J. Syst. Bacteriol. 27:75-85.

18. Silcox, V. A., R. C. Good, and M. M. Floyd. 1981. Identification of clinically significant Mycobacterium fortuitum complex isolates. J. Clin. Microbiol. 14:686-691.

19. Skerman, V. B. D., V. McGowan, and P. H. A. Sneath (ed.). 1980. Approved lists of bacterial names. Int. J. Syst. Bacteriol. 30:225-420.

20. Sokal, R. R., and P. H. A. Sneath. 1963. Principles of numerical taxonomy. W. H. Freeman \& Co., San Francisco.

21. Tsukamura, M. 1967. Identification of mycobacteria. Tubercle 48:311-338.

22. Vestal, A. 1975. Procedures for the isolation and identification of mycobacteria. Publication (CDC) 76-8230. Centers for Disease Control, Atlanta, Ga. 